\title{
Relationship of salusin-alpha and salusin-beta levels with atherosclerosis in patients undergoing haemodialysis
}

Savas Sipahi ${ }^{1}, \mathrm{MD}$, Ahmed Bilal $\underline{\text { Genc }}^{2}, \mathrm{MD}$, Seyyid Bilal $\underline{\text { Acikgoz }^{2}}$, MD, Mehmet Yildirim $^{2}$, MD, Yakup Ersel $\underline{\text { Aksoy }}{ }^{3}$, MD, Mehmet Bulent Vatan ${ }^{4}$, MD, Hamad Dheir ${ }^{1}, \mathrm{MD}$, Mustafa Altındis ${ }^{5}$, MD

\begin{abstract}
INTRODUCTION In patients undergoing haemodialysis, cardiovascular mortality and morbidity, characterised by accelerated atherosclerosis and increased inflammation, are elevated. Salusins are newly defined molecules in the atherosclerotic processes, and while salusin-alpha (Sal- $\alpha$ ) acts as an antiatherogenic factor, salusin-beta (Sal- $\beta$ ) has a proatherogenic role. Their roles are as yet undefined in patients undergoing haemodialysis.

METHODS In this cross-sectional study, salusin levels, carotid intima-media thickness (CIMT) from the common carotid artery and pulse wave velocity (PWV) were measured for 180 patients undergoing haemodialysis and 90 healthy controls. RESULTS Mean Sal- $\alpha$ and Sal- $\beta$ levels in patients undergoing haemodialysis (Sal- $\alpha$ : $726.4 \pm 578.7$ pg/mL; Sal- $\beta$ : $1,080.4 \pm$ $757.1 \mathrm{pg} / \mathrm{mL}$ ) and healthy controls (Sal- $\alpha: 325.8 \pm 303.7 \mathrm{pg} / \mathrm{mL}$; Sal- $\beta$ : $268.1 \pm 409.0 \mathrm{pg} / \mathrm{mL}$ ) were determined. Negative correlation was observed between Sal- $\alpha$ levels and CIMT (patients undergoing haemodialysis: $r=-0.330, p<0.0001$; healthy controls: $r=-0.223, p=0.035$ ) and PWV (patients undergoing haemodialysis: $r=-0.210, p=0.005$; healthy controls: $r=-0.378, p<0.0001$ ) in both groups. In patients undergoing haemodialysis, positive correlation was observed between Sal- $\beta /$ Sal- $\alpha$ ratio and CIMT $(r=0.190, p=0.012)$ and PWV $(r=0.155, p=0.041)$. On subgroup analysis, Sal- $\alpha$ levels were found to be low in patients with diabetes mellitus.

CONCLUSION Patients undergoing haemodialysis have higher Sal- $\beta$ and Sal- $\alpha$ levels, and their higher Sal- $\beta /$ Sal- $\alpha$ ratio, in comparison with healthy controls, might have cardiovascular risk implications.
\end{abstract}

Keywords: atherosclerosis, haemodialysis, salusin-alpha, salusin-beta

\section{INTRODUCTION}

In patients undergoing haemodialysis treatment for end-stage renal disease (ESRD), cardiovascular mortality is highly increased when compared to the general population. ${ }^{(1)}$ In this patient group, high cardiovascular mortality is associated with accelerated atherosclerosis. ${ }^{(2)}$

In 2003, salusins were defined as a new class of vasoactive peptides by Shichiri et al. ${ }^{(3)}$ Salusin-alpha (Sal- $\alpha$ ) and salusinbeta (Sal- $\beta$ ) are peptides consisting of 28 and 20 amino acids, respectively. Preprosalusin is greatly released from vascular smooth muscle cells and endothelial cells. ${ }^{(3)}$ Salusins are synthesised and expressed from the renal system, central nervous system and vascular system of human, rat and mouse tissues. ${ }^{(4)}$ While salusins are expressed from coronary atherosclerotic plaques, expression of Sal- $\alpha$ is observed at a lower ratio when compared to that of Sal- $\beta{ }^{(5)}$ In an in vivo study, it was revealed that Sal- $\beta$ was synthesised by fibroblasts in the vascular smooth muscle cells of the media layer and by the media layer of aorta during coronary artery bypass graft surgeries in patients with coronary artery disease. ${ }^{(6)}$ Salusins have various effects on haemodynamic activities. ${ }^{(7)}$

It has been suggested that salusins play a role in the formation and progression of atherosclerosis. ${ }^{(8)}$ Sal- $\beta$ stimulates the expression of monocyte chemoattractant protein 1, interleukin-1 beta, nicotinamide adenine dinucleotide phosphate oxidase 2 and vascular cell adhesion molecule- 1 in endothelial cells. Thus, it facilitates monocyte adhesion in endothelial cells. ${ }^{(9)}$ Also, it increases the conversion of macrophages in the foam cells and expression of acylcoenzyme A transferase 1 (ACAT-1). ${ }^{(5)}$ Sal- $\beta$ stimulates vascular smooth muscle cells and fibroblast proliferation. ${ }^{(3)}$ Owing to these effects, it shows a proatherogenic effect. On the other hand, Sal- $\alpha$ shows an antiatherogenic effect by reducing macrophage foam cell formation and ACAT-1 expression. ${ }^{(5)}$

Atherosclerotic changes occurring in the carotid artery reflect atherosclerosis in the whole body, and it is accepted as the precursor of cardiovascular morbidity and mortality in the general population and ESRD patients. ${ }^{(10,11)}$ Determination of the changes in the artery wall via carotid intima-media thickness (CIMT) measurement using B-mode ultrasonography is accepted as a cheap, reliable and repeatable method..$^{(12,13)}$

Arterial stiffness and haemodynamic results related to this are independent indicators of poor cardiovascular prognosis and end-organ damage development. ${ }^{(14,15)}$ Noninvasive analysis of peripheral flow signals is commonly used in the assessment of arterial stiffness. ${ }^{(16,17)}$ Today, there are devices that automatically measure pulse wave velocity (PWV). ${ }^{(18)}$ In recent times, brachial cuff-based ambulatory oscillometric devices have been used

${ }^{1}$ Department of Internal Medicine, Division of Nephrology, ${ }^{2}$ Department of Internal Medicine, ${ }^{3}$ Department of Radiology, ${ }^{4}$ Department of Cardiology, ${ }^{5}$ Department of Microbiology, Sakarya University Faculty of Medicine, Sakarya, Turkey

Correspondence: Dr Savas Sipahi, Associate Professor, Department of Internal Medicine, Division of Nephrology, Sakarya University Faculty of Medicine, Adnan Menderes Cd. Saglik Sok. No: 195, 54100 Adapazari/Sakarya, Turkey. ssipahi@sakarya.edu.tr 
to efficiently analyse PWV. Augmentation pressure is taken to represent the difference between the second and first systolic peaks of the central pressure waveform. Augmentation index is defined as augmentation pressure expressed as a percentage of pulse pressure.

To the best of our knowledge, there is no information regarding the Sal- $\alpha$ and Sal- $\beta$ levels in patients undergoing haemodialysis. We aimed to define the relationship between CIMT and PWV - well-known atherosclerosis indicators - and salusin levels, and determine the factors affecting this relationship.

\section{METHODS}

This study was conducted in accordance with the Declaration of Helsinki. All participants provided written informed consent for the study, which was approved by the ethics committee of Sakarya University Faculty of Medicine, Sakarya, Turkey.

In total, 180 patients undergoing haemodialysis and 90 healthy controls were included in the study. The test group consisted of patients who had undergone haemodialysis treatment three days and four hours weekly for at least three months and were in the age range of 18-80 years. Control participants were healthy adults who were in a similar age group, had no chronic disease and did not have any episodes of infectious diseases within the previous three months. Patients with temporary renal dysfunction, active infectious/inflammatory episodes or acute ischaemic vascular disease histories within the previous three months, additional diseases causing chronic inflammation, chronic liver disease, positive hepatitis serology and those who did not provide written consent were excluded from the study.

Demographic (e.g. gender, date of birth, start date of haemodialysis, reasons for chronic renal insufficiency, presence of comorbid diseases, height, weight, hepatitis serology and medical treatment) and laboratory (e.g. complete blood count, C-reactive protein [CRP], ferritin, glycaemia, urea, creatinine, sodium, potassium, calcium, phosphorus, uric acid, total protein, albumin, alanine aminotransferase $[A L T]$, aspartate aminotransferase [AST], alkaline phosphatase [ALP], bicarbonate, haemoglobin A1c $[\mathrm{HbA1c}]$, lipid profile, intact parathyroid hormone [PTH] and $\mathrm{Kt} / \mathrm{V}$ for haemodialysis kinetics) data were determined for healthy controls and patients undergoing haemodialysis. For patients undergoing haemodialysis, while blood samples were taken before the midweek haemodialysis session, an additional tube of blood sample was withdrawn and maintained at $-80^{\circ} \mathrm{C}$ in order to examine salusin levels. Fasting blood samples of healthy controls were also taken and kept at $-80^{\circ} \mathrm{C}$.

The complete blood count, CRP, ferritin, glycaemia, urea, creatinine, sodium, potassium, calcium, phosphorus, uric acid, total protein, albumin, ALT, AST, ALP, HbA1c, lipid profile, PTH, total cholesterol, high-density lipoprotein cholesterol, triglycerides and bicarbonate were measured using a central laboratory biochemistry analyser (integrated chip technology ${ }^{\mathrm{TM}}$ for ion-selective electrode measurements; ARCHITECT c16000; Abbott Laboratories, Abbott Park, IL, USA).

Serum samples collected for Sal- $\alpha$ and Sal- $\beta$ levels were stored at $-80^{\circ} \mathrm{C}$. Human Sal- $\alpha$ enzyme-linked immunosorbent assay (ELISA) kit (Hangzhou Eastbiopharm Co Ltd, Hangzhou, Zhejiang, China) and human Sal- $\beta$ ELISA kit (Hangzhou Eastbiopharm Co Ltd) were used to determine Sal- $\alpha$ and Sal- $\beta$ levels. According to the manufacturer's instructions, $40-\mu \mathrm{L}$ serum samples from patients undergoing haemodialysis and healthy controls were used and the test was performed using the microELISA method. Test results were read using the Triturus (Grifols) ELISA reader (Grifols International, Barcelona, Catalonia, Spain) at $450-\mathrm{nm}$ wavelengths, and Sal- $\alpha$ and Sal- $\beta$ levels were determined. Sensitivity for Sal- $\alpha$ was $0.51 \mathrm{pg} / \mathrm{mL}$ (assay range: $5-1,000 \mathrm{pg} / \mathrm{mL}$ ) and sensitivity for Sal- $\beta$ was $5.22 \mathrm{pg} / \mathrm{mL}$ (assay range: $510-1,800 \mathrm{pg} / \mathrm{mL}$ ).

CIMT measurement was performed via B-mode examination using the ToshibaAplio 400 high-level colour Doppler ultrasonography device (Toshiba Medical Systems Corporation, Minato, Tokyo, Japan) and a linear probe with high-resolution central frequency of 7.5 (range 5-12) $\mathrm{mHz}$. It was performed by a single radiologist while giving approximately $20^{\circ}-45^{\circ}$ angle towards the neck with the patient lying in a supine position. In the proximal $(1 \mathrm{~cm}$ after bifurcation), medium $(4 \mathrm{~cm}$ after bifurcation) and distal (first $1 \mathrm{~cm}$ of bulbus) sections of the common carotid artery, intima-media thickness from the posterior wall was measured and the arithmetical mean of these three measurements taken. Assessments were planned for locations with laminar flow and not for locations where large plaques were present or turbulent flow was determined. According to the planned measurement type, left and right CIMTs were determined and their mean was recorded as the CIMT measurement.

Measurements were done by using the ambulatory blood pressure monitorisation system (Mobil-O-Graph NG, IEM, Stolberg, North Rhine-Westphalia, Germany) that shows brachial oscillometric blood pressure waves. After the patients had rested for approximately 15 minutes, measurements were taken by connecting a cuff to the left brachial artery level with the patient in a sitting position.

Statistical analysis was performed using IBM SPSS Statistics version 21.0 (IBM Corp, Armonk, NY, USA). During data assessment, the Kolmogorov-Smirnov test was used to determine whether variables had a normal distribution. Central distribution criteria of normally distributed numerical variables were specified as mean \pm standard deviation and numerical variables not showing normal distribution, were specified as median (range). Categorical data was expressed as percentage values. Student's t-test was used for comparing between-group differences of numerical variables with normal distribution while Mann-Whitney $U$ test was used for comparing between-groups differences of numerical variables not showing normal distribution. Chi-square test was used to assess categorical variables. Spearman's rank correlation analysis was used to assess the relationship between numerical variables not showing normal distribution. The reference of $r$ and comments were as follows: $0.00-0.19=$ very weak; $0.20-0.39=$ weak; $0.40-0.59=$ moderate; $0.60-0.79=$ strong; and $0.80-1.0=$ very strong. A p-value $<0.05$ was considered to be statistically significant. 
Table I. Demographic and biochemical characteristics of participants.

\begin{tabular}{|c|c|c|c|c|c|}
\hline \multirow[t]{2}{*}{ Variable } & \multicolumn{2}{|c|}{$\begin{array}{l}\text { Patients undergoing } \\
\text { haemodialysis }(n=180)\end{array}$} & \multicolumn{2}{|c|}{ Control group $(n=90)$} & \multirow[t]{2}{*}{ p-value } \\
\hline & Mean \pm SD & Median (range) & Mean \pm SD & Median (range) & \\
\hline Age (yr) & $56.6 \pm 12.9$ & $59.0(18-78)$ & $53.2 \pm 15.1$ & $50.5(24-80)$ & $0.033^{*}$ \\
\hline Body mass index $\left(\mathrm{kg} / \mathrm{m}^{2}\right)$ & $25.5 \pm 5.2$ & $25.1(15.7-43.6)$ & $26.5 \pm 4.2$ & $26.4(17.2-37.1)$ & $0.114^{+}$ \\
\hline Gender (no. [\%]) & & & & & $0.476^{\ddagger}$ \\
\hline Female & $64(35.6)$ & & $36(40.0)$ & & \\
\hline Smoking (no. of packets $\times$ yr) & $28.4 \pm 23.0$ & $20.0(0.2-105.0)$ & $22.9 \pm 24.9$ & $15.0(2.0-100.0)$ & $0.070^{*}$ \\
\hline Duration of haemodialysis (mth) & $64.4 \pm 52.1$ & $50.0(4.0-229.0)$ & & & \\
\hline Urea reduction ratio (\%) & $72.5 \pm 6.4$ & $73.4(52.0-91.0)$ & & & \\
\hline Mean blood pressure (mmHg) & $102.5 \pm 25.1$ & $98.0(59.0-184.0)$ & $95.7 \pm 16.8$ & $94.0(9.0-144.0)$ & $0.186^{*}$ \\
\hline Calcium (mg/dL) & $8.5 \pm 0.7$ & $8.6(6.8-10.8)$ & $9.3 \pm 0.2$ & $9.4(8.7-10.0)$ & $<0.001^{+}$ \\
\hline Phosphorus (mg/dL) & $4.9 \pm 1.2$ & $5.0(2.5-8.7)$ & $2.9 \pm 0.5$ & $3.0(2.0-4.7)$ & $<0.001^{*}$ \\
\hline Albumin (g/dL) & $4.0 \pm 0.3$ & $4.0(1.8-4.9)$ & $4.1 \pm 0.23$ & $4.2(3.5-4.7)$ & $<0.001^{*}$ \\
\hline Uric acid (mg/dL) & $6.4 \pm 1.1$ & $6.3(3.3-11.2)$ & $5.2 \pm 1.36$ & $5.2(0.3-8.0)$ & $<0.001^{*}$ \\
\hline C-reactive protein (mg/L) & $12.7 \pm 23.6$ & $6.6(0.4-275)$ & $4.4 \pm 3.5$ & $3.1(3.1-28.3)$ & $<0.001^{*}$ \\
\hline Alkaline phosphatase (U/L) & $175.8 \pm 108.5$ & $151(55-784)$ & $83.6 \pm 23.8$ & $78(35-145)$ & $<0.001^{*}$ \\
\hline Intact parathyroid hormone (pg/mL) & $623.2 \pm 517.5$ & $487.9(0.0-3,718.0)$ & $59.6 \pm 25.7$ & $55.9(20.3-166.3)$ & $<0.001^{*}$ \\
\hline Ferritin (ng/mL) & $502.7 \pm 266.6$ & $470(19-1,522)$ & $72.8 \pm 67.1$ & $52(4-329)$ & $0.000^{\dagger}$ \\
\hline Total cholesterol (mg/dL) & $165.3 \pm 36.6$ & $161(91-256)$ & $207.1 \pm 37.2$ & $202(130-287)$ & $<0.001^{*}$ \\
\hline Low-density lipoprotein cholesterol (mg/dL) & $96.8 \pm 33.4$ & $94(33-188)$ & $145.8 \pm 31.0$ & $145(91-224)$ & $<0.001^{\dagger}$ \\
\hline High-density lipoprotein cholesterol (mg/dL) & $38.9 \pm 11.8$ & $38(21-84)$ & $49.1 \pm 11.4$ & $48(28-78)$ & $<0.001^{*}$ \\
\hline Triglyceride (mg/dL) & $148.1 \pm 57.8$ & $152(33-461)$ & $137.3 \pm 60.8$ & $120(52-311)$ & $0.054^{*}$ \\
\hline Haemoglobin (g/dL) & $11.2 \pm 1.2$ & $11.2(8.0-15.0)$ & $13.8 \pm 1.5$ & $14.0(8.0-16.9)$ & $<0.001^{*}$ \\
\hline Haematocrit (\%) & $34.4 \pm 4.0$ & $34.7(23.8-46.4)$ & $41.5 \pm 4.3$ & $42.0(26.6-51.9)$ & $<0.001^{*}$ \\
\hline
\end{tabular}

P-value calculated using *Mann-Whitney $U$ test, $†$ Student's $t$-test and ¥chi-square test. SD: standard deviation

\section{RESULTS}

Table I shows the demographic characteristics and biochemical parameters of all participants. Patients undergoing haemodialysis were older than healthy controls $(p=0.033)$. Although there was no significant difference in terms of body mass index and gender, smoking was more prevalent among patients undergoing haemodialysis $(p=0.070)$. Among patients undergoing haemodialysis, diabetes mellitus $(n=58,32.2 \%)$ and hypertension ( $n=132,73.3 \%$ ) were present. Duration of haemodialysis among patients undergoing haemodialysis was $64.4 \pm 52.1$ months. There was correlation between the duration of haemodialysis and salusin levels of patients undergoing haemodialysis (Sal- $\alpha: r=0.279, p<0.0001$; Sal- $\beta: r=0.325$, $\mathrm{p}<0.0001$ ).

Table II shows the Sal- $\alpha$ and Sal- $\beta$ levels of healthy controls and patients undergoing haemodialysis. It was determined that both Sal- $\alpha$ and Sal- $\beta$ levels in patients undergoing haemodialysis were increased when compared to healthy controls (both $p<0.001)$. Similarly, the Sal- $\beta /$ Sal- $\alpha$ ratio was high among patients undergoing haemodialysis.

CIMT measurements between the two patient groups showed significant differences (patients undergoing haemodialysis: 0.92 $\pm 0.21 \mathrm{~mm}$ vs. healthy controls: $0.67 \pm 0.16 \mathrm{~mm} ; \mathrm{p}<0.0001$ ).
As expected, CIMT was thicker among patients undergoing haemodialysis.

Table III, which provides results of pulse wave analysis (PWA), shows that augmentation pressure $(p=0.007)$, augmentation index $(p=0.010)$ and PWV $(p<0.001)$ were higher among patients undergoing haemodialysis.

PWV was significantly higher for more patients undergoing haemodialysis than healthy controls $(n=64,35.6 \%$ vs. $n=12$; $13.3 \% ; p<0.0001)$. Although high augmentation index was also determined for more patients undergoing haemodialysis than healthy controls ( $n=32,17.8 \%$ vs. $n=11,12.2 \%$ ), this did not reach statistical significance $(p=0.240)$.

Negative correlation was found between Sal- $\alpha$ levels and CIMT in both patients undergoing haemodialysis $(r=-0.330, p<0.0001)$ and healthy controls $(r=-0.223, p=0.035)$. Similarly, there was negative correlation between Sal- $\alpha$ levels and PWV for both patients undergoing haemodialysis $(r=-0.210, p=0.005)$ and healthy controls $(r=-0.378, p<0.0001)$. No correlation was found between Sal- $\beta$ levels and PWV. There was positive correlation between Sal- $\beta$ / Sal- $\alpha$ ratio and CIMT $(r=0.190, p=0.012)$ and PWV $(r=0.155$, $p=0.041$ ) among patients undergoing haemodialysis.

In the subgroup analysis, where we compared patients undergoing haemodialysis with diabetes mellitus to those 
Table II. Salusin-alpha (Sal- $\alpha$ ) and salusin-beta (Sal- $\beta$ ) levels of participants.

\begin{tabular}{|c|c|c|c|c|c|}
\hline \multirow[t]{2}{*}{ Variable } & \multicolumn{2}{|c|}{ Patients undergoing haemodialysis $(n=174)$} & \multicolumn{2}{|c|}{ Control group $(n=90)$} & \multirow[t]{2}{*}{ p-value } \\
\hline & Mean \pm SD & Median (range) & Mean \pm SD & Median (range) & \\
\hline Sal-a (pg/mL) & $726.39 \pm 578.73$ & $729.05(2.00-1,982.57)$ & $325.78 \pm 303.66$ & $196.00(32.99-1,003.83)$ & $<0.001^{*}$ \\
\hline Sal- $\beta$ (pg/mL) & $1,080.40 \pm 757.13$ & $974.86(30.40-2,561.50)$ & $268.07 \pm 409.01$ & $25.88(6.88-1,513.45)$ & $<0.001^{*}$ \\
\hline Sal- $\beta /$ Sal-a ratio & $2.37 \pm 2.89$ & $2.02(0.03-24.30)$ & $0.46 \pm 0.51$ & $0.21(0.04-3.13)$ & $<0.001^{*}$ \\
\hline
\end{tabular}

*Mann-Whitney U test. SD: standard deviation

Table III. Results of pulse wave analysis of participants.

\begin{tabular}{|c|c|c|c|c|c|}
\hline \multirow[t]{2}{*}{ Variable } & \multicolumn{2}{|c|}{$\begin{array}{l}\text { Patients undergoing } \\
\text { haemodialysis }(n=180)\end{array}$} & \multicolumn{2}{|c|}{ Control group $(\mathbf{n}=90)$} & \multirow[t]{2}{*}{ p-value } \\
\hline & Mean \pm SD & Median (range) & Mean \pm SD & Median (range) & \\
\hline Augmentation index (\%) & $22.9 \pm 11.5$ & $23(1-53)$ & $19.2 \pm 11.7$ & $17(1-55)$ & $0.010^{*}$ \\
\hline Pulse wave velocity (m/s) & $8.8 \pm 1.1$ & $8.7(4.0-13.8)$ & $7.3 \pm 2.1$ & $6.6(4.5-13.3)$ & $<0.001^{\dagger}$ \\
\hline
\end{tabular}

P-value calculated using *Mann-Whitney $U$ test or $+S t$ dent's $t$-test. SD: standard deviation

without diabetes mellitus in terms of salusin levels, median Sal- $\alpha$ levels were determined as 337.8 (range $2.0-1,806.6$ ) $\mathrm{pg} / \mathrm{mL}$ for patients undergoing haemodialysis with diabetes mellitus and 754.6 (range $3.7-1,982.5$ ) $\mathrm{pg} / \mathrm{mL}$ for patients undergoing haemodialysis without diabetes mellitus. Median Sal- $\beta /$ Sal- $\alpha$ ratio was 2.2 (range 0.04-24.3) for patients undergoing haemodialysis with diabetes mellitus and 1.7 (range 0.03-19.8) in patients undergoing haemodialysis without diabetes mellitus. Among patients undergoing haemodialysis without diabetes mellitus, Sal- $\alpha$ levels were significantly higher $(p=0.003)$ and Sal- $\beta /$ Sal- $\alpha$ ratio was lower $(p=0.008)$.

\section{DISCUSSION}

In this study, we found that patients undergoing haemodialysis had higher Sal- $\alpha$ and Sal- $\beta$ levels when compared to healthy controls. Salusin levels were found to be higher in our study among healthy controls and patients undergoing haemodialysis than in previous studies. ${ }^{(19)}$ High salusin levels were anticipated among patients undergoing haemodialysis due to the characteristics of this patient group. However, we also found high salusin values among our healthy controls, and it is possible that this finding was related to the relatively older age of our patients. ${ }^{(19)}$ Spearman's rank correlation analysis showed correlation between age and salusin levels. Among patients undergoing haemodialysis, values could be determined only by diluting the serum for 45 patients with Sal- $\alpha$ level above $1,000 \mathrm{pg} / \mathrm{mL}$ and for 63 patients with Sal- $\beta$ values above $1,600 \mathrm{pg} / \mathrm{mL}$. Also, correlation was observed between salusin levels and the haemodialysis periods of patients.

In previous studies, the antiatherogenic peptide role of Sal- $\alpha$ was shown by Watanabe et al in patients with coronary atherosclerosis, as confirmed by angiography, and in hypertensive patients. ${ }^{(5,20)}$ Du et al indicated the correlation between coronary artery disease and Sal- $\alpha$ levels. ${ }^{(21)}$ The proatherogenic effect of Sal- $\beta$ has also been reported. ${ }^{(22)}$ However, to the best of our knowledge, no similar study in the published literature has evaluated patients undergoing haemodialysis. In Kimoto et al's study on patients with renal insufficiency, ${ }^{(23)}$ although it was emphasised that salusin levels were decreased as the glomerular filtration rate decreased, the authors did not provide any data regarding patients undergoing haemodialysis. The current study was performed on 180 patients undergoing haemodialysis in comparison with the study by Kimoto et al, which only involved 15 patients with estimated glomerular filtration rate $<15 / \mathrm{min} / 1.73 \mathrm{~m}^{2}$. Whether Sal- $\alpha$ levels would increase upon the commencement of haemodialysis among patients with ESRD is yet to be evaluated. ${ }^{(23)}$

In the present study, the median Sal- $\beta /$ Sal- $\alpha$ ratio was found to be different between patients undergoing haemodialysis (2.0 [range 0.03-24.3]) and healthy controls (0.21 [range 0.04-3.13]) $(p<0.001)$. This finding highlights the impaired/accelerated atherosclerotic process in patients undergoing haemodialysis. In previous studies by Watanabe et al, the opposing effects of Sal- $\alpha$ and Sal- $\beta$ on atherosclerosis have been indicated although the Sal- $\beta /$ Sal- $\alpha$ ratio was not emphasised. The low levels of Sal- $\alpha$ in hypertensive patients and patients with cardiac ischaemia have been reported previously. ${ }^{(5,20)}$

CIMT and PWA measurements, which are reliable indicators of atherosclerosis, were also significantly different between patients undergoing haemodialysis and healthy controls. CIMT was $0.92 \pm 0.21 \mathrm{~mm}$ and $0.67 \pm 0.16 \mathrm{~mm}$ among patients undergoing haemodialysis and healthy controls, respectively ( $p<0.0001)$. Likewise, among the PWA measurements, mean PWV (patients undergoing haemodialysis: $8.8 \pm 1.1 \mathrm{~m} / \mathrm{s}$; healthy controls: $7.3 \pm 2.1 \mathrm{~m} / \mathrm{s} ; \mathrm{p}<0.001$ ) and augmentation index (patients undergoing haemodialysis: $22.9 \% \pm 11.5 \%$; healthy controls: $19.2 \% \pm 11.7 \% ; p=0.01$ ) were significantly different between the two study groups.

On examining Sal- $\alpha$ and Sal- $\beta$ levels as well as the CIMT and PWA results, which are well-known atherosclerosis indicators, negative correlation was found between Sal- $\alpha$ levels and CIMT and PWA measurements for both patients undergoing haemodialysis and healthy controls. CIMT values among patients undergoing haemodialysis with high Sal- $\alpha$ levels were thinner and corresponding PWA measurements better. As shown in previous 
studies, this underscores the antiatherogenic characteristics of Sal- $\alpha$ in both our groups. ${ }^{(5,20)}$ Along similar lines, the Sal- $\beta /$ Sal- $\alpha$ ratio was also significant as an indicator of atherosclerosis in both groups.

During subgroup analysis, Sal- $\alpha$ levels were found to be lower in patients undergoing haemodialysis with diabetes mellitus and higher among those without diabetes mellitus. Correspondingly, the Sal- $\beta /$ Sal- $\alpha$ ratio was higher among patients undergoing haemodialysis with diabetes mellitus and lower in those without. In contrast, a study by Aydın et al on patients with gestational diabetes mellitus found that Sal- $\alpha$ and Sal- $\beta$ levels were lower in their patients. ${ }^{(24)}$

In a study by $\mathrm{Ti}$ et al on patients with essential hypertension, no significant difference was found in terms of Sal- $\alpha$ levels between hypertensive and nonhypertensive patients. ${ }^{(25)}$ However, in the present study, a weak positive correlation was found between diastolic blood pressure and Sal- $\beta$ levels among patients undergoing haemodialysis $(\mathrm{p}<0.05)$.

During subgroup analysis, the relationship between salusin levels and antihypertensive and antihyperlipidaemic drugs, which are the drug groups known to have effects on atherosclerosis, were examined. However, we did not find any correlation between the variables that might have affected treatment. Unlike our study, a previous study by Grzegorzewska et al on patients undergoing haemodialysis indicated that lipid-lowering treatment caused Sal- $\alpha$ expression. ${ }^{(26)}$

Our study thus indicated a correlation between Sal- $\alpha$ levels and atherosclerosis in both patients undergoing haemodialysis and healthy controls. However, there was no evidence of Sal- $\beta$ levels and atherosclerosis on comparable lines. It is possible that this was because atherosclerosis in patients undergoing haemodialysis may be caused by a number of other factors. The pathogenesis of cardiovascular damage in patients undergoing haemodialysis is complex. These patients carry both the classical risk factors of advanced atherosclerosis as well as risk factors secondary to chronic renal disease and the underlying disease. The role of increasing calcification stimulators (e.g. hyperphosphataemia, hypercalcaemia, increased oxidised low-density lipoprotein cholesterol and hyperleptinaemia) and decreasing calcification inhibitors (e.g. matrix Gla [gamma-carboxyglutamic acid] protein, fetuin, osteoprotegerin and osteopontin) has also been discussed. ${ }^{(27)}$ To the best of our knowledge, no study has as yet been conducted on the metabolism and excretion pathways of Sal- $\alpha$ and Sal- $\beta$. Therefore, although both Sal- $\alpha$ and Sal- $\beta$ were higher in patients with ESRD undergoing haemodialysis in comparison with healthy controls, we could not conclusively draw implications that they were good markers for cardiovascular risk. Extrapolating on previous experiences with the use of creatinine kinase and troponin in patients with renal disease, and especially among patients with ESRD, it is possible that different reference points might be needed for these parameters.

Nevertheless, Sal- $\beta /$ Sal- $\alpha$ ratio in patients undergoing haemodialysis and healthy controls is an important finding of our study; so is the low Sal- $\alpha$ levels found in patients undergoing haemodialysis with diabetes mellitus. However, we were unable to find any relationship between the variables studied and the treatment provided to our patients.

As higher Sal- $\alpha$ and Sal- $\beta$ levels were identified among patients undergoing haemodialysis with ESRD, these new reference points could potentially serve to identify patients at higher risk of cardiovascular events. A longitudinal study is warranted to determine their significance vis-à-vis the traditional cardiovascular risk markers.

\section{ACKNOWLEDGEMENT}

This study was funded by TUBi TAK (The Scientific and Technological Research Council of Turkey; Project no.: 215S173/2016).

\section{REFERENCES}

1. Sarnak MJ, Levey AS. Cardiovascular disease and chronic renal disease: a new paradigm. Am J Kidney Dis 2000; 35(4 Suppl 1):S117-31.

2. Baigent $\mathrm{C}$, Burbury $\mathrm{K}$, Wheeler D. Premature cardiovascular disease in chronic renal failure. Lancet 2000; 356:147-52.

3. Shichiri M, Ishimaru S, Ota T, et al. Salusins: newly identified bioactive peptides with hemodynamic and mitogenic activities. Nat Med 2003; 9:1166-72.

4. Suzuki N, Shichiri M, Akashi T, et al. Systemic distribution of salusin expression in the rat. Hypertens Res 2007; 30:1255-62.

5. Watanabe T, Nishio K, Kanome T, et al. Impact of salusin-alpha and -beta on human macrophage foam cell formation and coronary atherosclerosis. Circulation 2008; 117:638-48

6. Aydin S, Eren MN, Aydin S, Ozercan IH, Dagli AF. The bioactive peptides salusins and apelin-36 are produced in human arterial and venous tissues and the changes of their levels during cardiopulmonary bypass. Peptides 2012; 37:233-9.

7. Chen WW, Sun HJ, Zhang F, et al. Salusin- $\beta$ in paraventricular nucleus increases blood pressure and sympathetic outflow via vasopressin in hypertensive rats. Cardiovasc Res 2013; 98:344-51.

8. Zhou $\mathrm{CH}$, Liu L, Liu L, et al. Salusin- $\beta$ not salusin- $\alpha$ promotes vascular inflammation in ApoE-deficient mice via the $\mathrm{I}-\kappa \mathrm{B} \alpha / \mathrm{NF}-\kappa \mathrm{B}$ pathway. PLoS One 2014; 9:e91468.

9. Koya T, Miyazaki T, Watanabe $T$, et al. Salusin- $\beta$ accelerates inflammatory responses in vascular endothelial cells via NF- $\mathrm{KB}$ signaling in LDL receptordeficient mice in vivo and HUVECs in vitro. Am J Physiol Heart Circ Physiol 2012; 303:H96-105.

10. Zoccali C, Benedetto FA, Maas R, et al. Asymmetric dimethylarginine, C-reactive protein, and carotid intima-media thickness in end-stage renal disease. J Am Soc Nephrol 2002; 13:490-6.

11. Nishizawa Y, Shoji T, Maekawa K, et al. Intima-media thickness of carotid artery predicts cardiovascular mortality in hemodialysis patients. Am J Kidney Dis 2003; 41(3 Suppl 1):S76-9.

12. Kuller L, Borhani N, Furberg C, et al. Prevalence of subclinical atherosclerosis and cardiovascular disease and association with risk factors in the Cardiovascular Health Study. Am J Epidemiol 1994; 139:1164-79.

13. Touboul PJ, Hennerici MG, Meairs S, et al. Mannheim carotid intima-media thickness consensus (2004-2006). An update on behalf of the Advisory Board of the 3rd and 4th Watching the Risk Symposium, 13th and 15th European Stroke Conferences, Mannheim, Germany, 2004, and Brussels, Belgium, 2006. Cerebrovasc Dis 2007; 23:75-80.

14. Vlachopoulos C, Aznaouridis K, Stefanadis C. Prediction of cardiovascular events and all-cause mortality with arterial stiffness: a systematic review and meta-analysis. J Am Coll Cardiol 2010; 55:1318-27.

15. Laurent S, Cockcroft J, Van Bortel L, et al; European Network for Non-invasive Investigation of Large Arteries. Expert consensus document on arterial stiffness: methodological issues and clinical applications. Eur Heart J 2006; 27:2588-605.

16. Mancia G, Fagard R, Narkiewicz K, et al; Task Force Members. 2013 ESH/ESC Guidelines for the management of arterial hypertension: the Task Force for the management of arterial hypertension of the European Society of Hypertension (ESH) and of the European Society of Cardiology (ESC). J Hypertens 2013; 31:1281-357.

17. Mitchell GF, Hwang SJ, Vasan RS, et al. Arterial stiffness and cardiovascular events: the Framingham Heart Study. Circulation 2010; 121:505-11.

18. Rajzer MW, Wojciechowska W, Klocek M, et al. Comparison of aortic pulse wave velocity measured by three techniques: Complior, SphygmoCor and Arteriograph. J Hypertens 2008; 26:2001-7.

19. Kołakowska U, Kuroczycka-Saniutycz E, Wasilewska A, Olański W. Is the 
serum level of salusin- $\beta$ associated with hypertension and atherosclerosis in the pediatric population? Pediatr Nephrol 2015; 30:523-31.

20. Watanabe T, Sato K, Itoh F, et al. The roles of salusins in atherosclerosis and related cardiovascular diseases. J Am Soc Hypertens 2011; 5:359-65.

21. Du SL, Wang WJ, Wan J, et al. Serum salusin- $\alpha$ levels are inversely correlated with the presence and severity of coronary artery disease. Scand J Clin Lab Invest 2013; 73:339-43.

22. Nakayama C, Shichiri M, Sato K, Hirata Y. Expression of prosalusin in human neuroblastoma cells. Peptides 2009; 30:1362-7.

23. Kimoto S, Sato K, Watanabe T, et al. Serum levels and urinary excretion of salusin-alpha in renal insufficiency. Regul Pept 2010; 162:129-32.

24. Aydin S, Celik O, Gurates B, et al. Concentrations of preptin, salusins and hepcidins in plasma and milk of lactating women with or without gestational diabetes mellitus. Peptides 2013; 49:123-30.

25. Ti Y, Wang F, Wang ZH, et al. Associations of serum salusin-alpha levels with atherosclerosis and left ventricular diastolic dysfunction in essential hypertension. J Hum Hypertens 2012; 26:603-9.

26. Grzegorzewska AE, Niepolski L, Sikora J, et al. Effect of lifestyle changes and atorvastatin administration on dyslipidemia in hemodialysis patients: a prospective study. Pol Arch Med Wewn 2014; 124:443-51.

27. Liabeuf S, Okazaki H, Desjardins L, et al. Vascular calcification in chronic kidney disease: are biomarkers useful for probing the pathobiology and the health risks of this process in the clinical scenario? Nephrol Dial Transplant 2014; 29:1275-84. 